\title{
The brick polytope of a sorting network
}

\author{
Vincent Pilaud ${ }^{1}$ and Francisco Santos ${ }^{2}$ \\ ${ }^{1}$ Université Paris Diderot, Paris, France \\ ${ }^{2}$ Universidad de Cantabria, Santander, Spain
}

\begin{abstract}
The associahedron is a polytope whose graph is the graph of flips on triangulations of a convex polygon. Pseudotriangulations and multitriangulations generalize triangulations in two different ways, which have been unified by Pilaud and Pocchiola in their study of pseudoline arrangements with contacts supported by a given network. In this paper, we construct the "brick polytope" of a network, obtained as the convex hull of the "brick vectors" associated to each pseudoline arrangement supported by the network. We characterize its vertices, describe its faces, and decompose it as a Minkowski sum of simpler polytopes. Our brick polytopes include Hohlweg and Lange's many realizations of the associahedron, which arise as brick polytopes of certain well-chosen networks.

Résumé. L'associaèdre est un polytope dont le graphe est le graphe des flips sur les triangulations d'un polygone convexe. Les pseudotriangulations et les multitriangulations généralisent les triangulations dans deux directions différentes, qui ont été unifiées par Pilaud et Pocchiola au travers de leur étude des arrangements de pseudodroites avec contacts couvrant un support donné. Nous construisons ici le "polytope de briques" d'un support, obtenu comme l'enveloppe convexe des "vecteurs de briques" associés à chaque arrangement de pseudodroites couvrant ce support. Nous caractérisons les sommets de ce polytope, décrivons ses faces et le décomposons en somme de Minkowski de polytopes élémentaires. Notre construction contient toutes les réalisations de l'associaèdre d'Hohlweg et Lange, qui apparaissent comme polytopes de briques de certains supports bien choisis.
\end{abstract}

Keywords: associahedron, sorting networks, pseudoline arrangements with contacts

\section{Introduction}

This paper focusses on polytopes realizing flip graphs on certain geometric and combinatorial structures. The motivating example is the associahedron whose vertices correspond to triangulations of a convex polygon and whose edges correspond to flips between them - see Figure 5. The associahedron appears under various motivations ranging from geometric combinatorics to algebra, and several different constructions have been proposed by Lee (1989), Gel'fand et al. (1994), Billera et al. (1990), Loday (2004), and Hohlweg and Lange (2007). See the book of De Loera et al. (2010) about triangulations of point sets.

In their study of the graph of flips on pseudoline arrangements with contacts supported by a given network, Pilaud and Pocchiola (2010) unified two different generalizations of triangulations of convex polygons: pseudotriangulations of planar point sets (see Rote et al. (2008)) and multitriangulations of convex polygons (see Pilaud and Santos (2009) or Pilaud (2010)). Our paper is based on this framework, and we recall its definitions and basic properties in Section 2

\footnotetext{
${ }^{\dagger}$ Research supported by grant MTM2008-04699-C03-02 of the Spanish Ministry of Education and Science. 1365-8050 @ 2011 Discrete Mathematics and Theoretical Computer Science (DMTCS), Nancy, France
} 
In this paper, we define and study the brick polytope of a network $\mathcal{N}$, obtained as the convex hull of vectors associated to each pseudoline arrangement supported by $\mathcal{N}$. Our main result is the characterization of the pseudoline arrangements which give rise to the vertices of the brick polytope of $\mathcal{N}$, from which we derive a combinatorial description of its faces. We furthermore provide a natural decomposition of the brick polytope into a Minkowski sum of simpler polytopes. These results are presented in Section 3 .

We observe in Section 4 that our brick polytopes coincide (up to translation) with the associahedra of Hohlweg and Lange (2007) for certain well-chosen networks. We thus provide a different point of view and complete the combinatorial description of their associahedra. As a supplementary motivation of our construction, let us also mentioned that permutahedra and cyclohedra naturally show up in our setting.

\section{The brick polytope of a sorting network}

\subsection{Sorting networks, pseudoline arrangements, and flips}

A network $\mathcal{N}$ is a set of $n$ horizontal lines (called levels), together with $m$ vertical segments (called commutators) joining two consecutive horizontal lines, such that no two commutators have a common endpoint - see Figure 1 (left). The bricks of $\mathcal{N}$ are its $m-n+1$ bounded cells.

A pseudoline is an abscissa monotone path on $\mathcal{N}$ which starts at a level $\ell$ and ends at the level $n+1-\ell$. A contact between two pseudolines is a commutator whose endpoints are contained one in each pseudoline, and a crossing between two pseudolines is a commutator traversed by both pseudolines. A pseudoline arrangement (with contacts) is a set of $n$ pseudolines supported by $\mathcal{N}$ such that any two of them have precisely one crossing, some (perhaps zero) contacts, and no other intersection - see Figure 1 (middle and right). Observe that a pseudoline arrangement supported by $\mathcal{N}$ is completely determined by its $\left(\begin{array}{l}n \\ 2\end{array}\right)$ crossings, or equivalently by its $m-\left(\begin{array}{l}n \\ 2\end{array}\right)$ contacts. We say that a network is sorting when it supports some pseudoline arrangements. We denote by $\operatorname{Arr}(\mathcal{N})$ the set of pseudoline arrangements supported by $\mathcal{N}$.

There is a natural flip operation which transforms a pseudoline arrangement supported by $\mathcal{N}$ into another one by exchanging the position of a contact. More precisely, if $V$ is the set of contacts of a pseudoline arrangement $\Lambda$ supported by $\mathcal{N}$, and if $v \in V$ is a contact between two pseudolines of $\Lambda$ which cross at $w$, then $V \triangle\{v, w\}$ is the set of contacts of another pseudoline arrangement supported by $\mathcal{N}-$ see Figure 1 (middle and right). The graph of flips $G(\mathcal{N})$ is the graph whose nodes are the pseudoline arrangements supported by $\mathcal{N}$ and whose edges are the flips between them. This graph was introduced and studied by Pilaud and Pocchiola (2010), whose first statement is the following result:
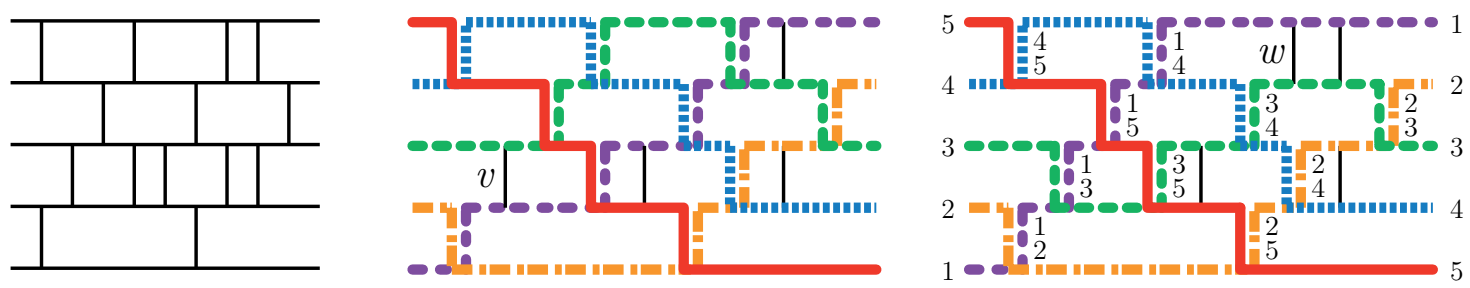

Fig. 1: A sorting network $\mathcal{N}$ (left) and two pseudoline arrangements supported by $\mathcal{N}$ and related by a flip (right). The rightmost pseudoline arrangement is the greedy pseudoline arrangement $\Gamma(\mathcal{N})$, whose flips are all increasing. It is obtained by sorting the permutation $(5,4,3,2,1)$ according to the network $\mathcal{N}$. 
Theorem 2.1 (Pilaud and Pocchiola (2010)) The graph of flips $G(\mathcal{N})$ of a sorting network $\mathcal{N}$ with $n$ levels and $m$ commutators is $\left(m-\left(\begin{array}{c}n \\ 2\end{array}\right)\right)$-regular and connected.

Regularity of the graph of flips is obvious since every contact induces a flip. For the connectivity, define a flip to be increasing if the added contact lies on the left of the removed contact. The oriented graph of increasing flips is clearly acyclic and is proved to have a unique source by Pilaud and Pocchiola (2010) (and thus, to be connected). This source is called the greedy pseudoline arrangement supported by $\overline{\mathcal{N}}$ and is denoted by $\Gamma(\mathcal{N})$. It is characterized by the property that any of its contacts is located to the right of its corresponding crossing. It can be computed by sorting the permutation $(n, n-1, \ldots, 2,1)$ according to the sorting network $\mathcal{N}$ - see Figure 1 (right). We will use this pseudoline arrangement later. We refer to Pilaud and Pocchiola (2010) for further details and applications of the greedy pseudoline arrangement.

Theorem 2.1 is a motivation to consider the simplicial complex $\Delta(\mathcal{N})$ whose maximal simplices are the sets of contacts of pseudoline arrangements supported by $\mathcal{N}$. As a particular subword complex of Knutson and Miller (2004), this complex $\Delta(\mathcal{N})$ is known to form a combinatorial sphere, but it remains open to know whether it is the boundary complex of a $\left(m-\left(\begin{array}{l}n \\ 2\end{array}\right)\right)$-dimensional simplicial polytope. In this article, we construct a polytope whose graph is a subgraph of $G(\mathcal{N})$. For certain networks (see Definition 3.4, this subgraph is even the whole graph $G(\mathcal{N})$, and we obtain a polytope whose boundary complex is $\Delta(\mathcal{N})$.

\subsection{The brick polytope}

Definition 2.2 Let $\mathcal{N}$ be a sorting network with n levels. The brick vector of a pseudoline arrangement $\Lambda$ supported by $\mathcal{N}$ is the vector $\omega(\Lambda) \in \mathbb{R}^{n}$ whose ith coordinate is the number of bricks of $\mathcal{N}$ located below the ith pseudoline of $\Lambda$ (the one which starts at level $i$ and finishes at level $n+1-i$ ). The brick polytope $\Omega(\mathcal{N}) \subset \mathbb{R}^{n}$ of the sorting network $\mathcal{N}$ is the convex hull of the brick vectors of all pseudoline arrangements supported by $\mathcal{N}$.

This article describes the combinatorial properties of the brick polytope $\Omega(\mathcal{N})$ in terms of the properties of the supporting network $\mathcal{N}$. In Section 3 , we provide a characterization of the pseudoline arrangements supported by $\mathcal{N}$ whose brick vectors are vertices of $\Omega(\mathcal{N})$, from which we derive a description of the faces of $\Omega(\mathcal{N})$. We also provide a natural decomposition of $\Omega(\mathcal{N})$ into a Minkowski sum of simpler polytopes.

We start by observing that the brick polytope is not full dimensional. Define the depth of a brick of $\mathcal{N}$ to be the number of levels located above it, and let $D(\mathcal{N})$ be the sum of the depths of all the bricks of $\mathcal{N}$. Since any pseudoline arrangement supported by $\mathcal{N}$ covers each brick as many times as its depth, all brick vectors are contained in the following hyperplane:

Lemma 2.3 The brick polytope $\Omega(\mathcal{N})$ is contained in the hyperplane of equation $\sum_{i=1}^{n} x_{i}=D(\mathcal{N})$.

Example 2.4 (2-levels networks) Consider the network $\mathcal{X}_{m}$ formed by two levels related by $m$ commutators. Choosing the ith commutator of $\mathcal{X}_{m}$ as unique crossing, we obtain a pseudoline arrangement whose brick vector is $(m-i, i-1)$. The graph of flips is the complete graph on $m$ vertices while the brick polytope $\Omega\left(\mathcal{X}_{m}\right)$ is a segment contained in the hyperplane of equation $x+y=m-1$.
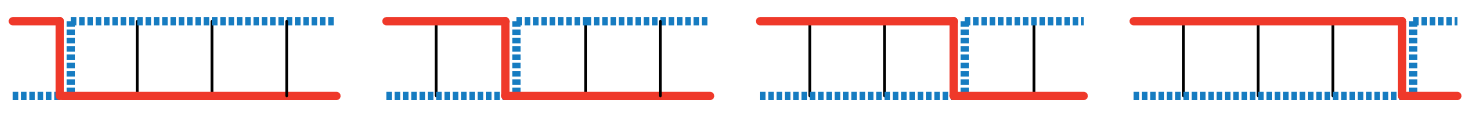

Fig. 2: The four pseudoline arrangements supported by the network $\mathcal{X}_{4}$ with two levels and four commutators. 


\section{Combinatorial description of the brick polytope}

In this section, we provide a combinatorial description of the face structure of the brick polytope $\Omega(\mathcal{N})$. For this purpose, we study the cone of the brick polytope $\Omega(\mathcal{N})$ at the brick vector of a given pseudoline arrangement supported by $\mathcal{N}$. Our main tool is the incidence configuration of the contact graph of a pseudoline arrangement, which we define next.

\subsection{The contact graph of a pseudoline arrangement}

Let $\mathcal{N}$ be a sorting network with $n$ levels and $m$ commutators, supporting a pseudoline arrangement $\Lambda$.

Definition 3.1 The contact graph of $\Lambda$ is the directed multigraph $\Lambda^{\#}$ with a node for each pseudoline of $\Lambda$ and an arc for each contact of $\Lambda$ oriented from the pseudoline passing above the contact to the pseudoline passing below it.
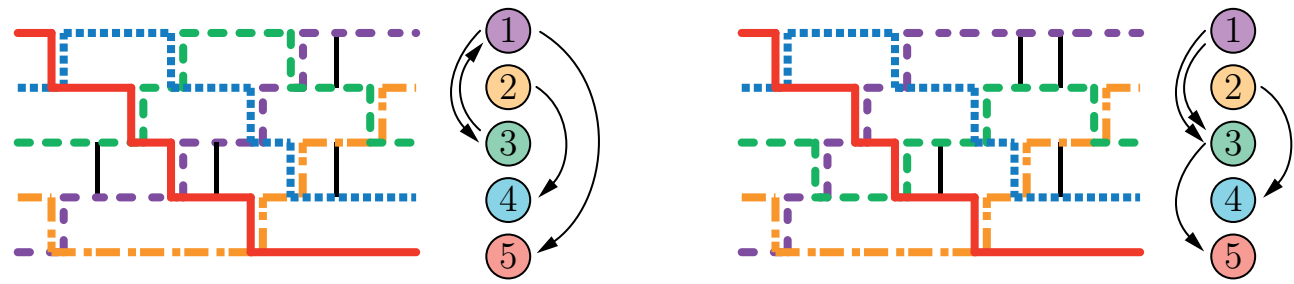

Fig. 3: The contact graphs of the pseudoline arrangements of Figure 1 Connected components are preserved by flip.

Let $\Lambda$ and $\Lambda^{\prime}$ denote two pseudoline arrangements with support $\mathcal{N}$ and related by a flip involving their $i$ th and $j$ th pseudolines - see Figure 3 . Then the directed multigraphs obtained by merging the vertices $i$ and $j$ in the contact graphs $\Lambda^{\#}$ and $\Lambda^{\prime \#}$ coincide. In particular, a flip preserves the connected components of the contact graph. Since the flip graph $G(\mathcal{N})$ is connected (Theorem 2.1, we obtain:

Lemma 3.2 The contact graphs of all pseudoline arrangements supported by $\mathcal{N}$ have the same connected components.

We say that a network is irreducible if the contact graphs of the pseudoline arrangements it supports are connected. It is easy to see that any sorting network can be decomposed into irreducible sorting networks:

Proposition 3.3 If a sorting network $\mathcal{N}$ supports pseudoline arrangements whose contact graphs have $p$ connected components, then it can be decomposed into p irreducible sorting networks $\mathcal{N}_{1}, \ldots, \mathcal{N}_{p}$ such that $\Delta(\mathcal{N})$ is the join $\Delta\left(\mathcal{N}_{1}\right) * \cdots * \Delta\left(\mathcal{N}_{p}\right)$ and $\Omega(\mathcal{N})$ is a translate of the product $\Omega\left(\mathcal{N}_{1}\right) \times \cdots \times \Omega\left(\mathcal{N}_{p}\right)$.

This proposition allow us to restrict our attention to irreducible sorting networks. Under this assumption, the following sorting networks have the fewest commutators:

Definition 3.4 An irreducible sorting network $\mathcal{N}$ is minimal if the following equivalent conditions hold:

- $\mathcal{N}$ has $n$ levels and $m=\left(\begin{array}{l}n \\ 2\end{array}\right)+n-1$ commutators.

- The contact graph of a pseudoline arrangement supported by $\mathcal{N}$ is a tree.

- The contact graphs of all pseudoline arrangements supported by $\mathcal{N}$ are trees.

Minimal networks naturally show up in Section 3.3 since their brick polytopes are of particular interest. 


\subsection{The incidence cone of a directed multigraph}

Let $G$ be a directed multigraph on $n$ vertices, whose underlying undirected graph is connected. We denote by $\left(e_{1}, \ldots, e_{n}\right)$ the canonical basis of $\mathbb{R}^{n}$ and $\mathbb{1}:=\sum e_{i}$.

Definition 3.5 The incidence configuration of $G$ is the set $I(G):=\left\{e_{j}-e_{i} \mid(i, j) \in G\right\} \subset \mathbb{R}^{n}$ of column vectors of its incidence matrix. The incidence cone of $G$ is the cone $C(G) \subset \mathbb{R}^{n}$ generated by $I(G)$.

Note that the incidence cone is contained in the linear subspace $\langle\mathbb{1} \mid x\rangle=0$. There is a correspondence between the graph properties of $G$ and the orientation properties of $I(G)$ - see Björner et al. (1999):

Remark 3.6 The vectors of the incidence configuration $I(H)$ of a subgraph $H$ of $G$

1. are independent if and only if $H$ has no (non-necessarily oriented) cycle, that is, if $H$ is a forest;

2. span the hyperplane $\langle\mathbb{1} \mid x\rangle=0$ if and only if $H$ is connected and spanning;

3. form a basis of the hyperplane $\langle\mathbb{1} \mid x\rangle=0$ if and only if $H$ is a spanning tree of $G$;

4. form a circuit if and only if $H$ is a (non-necessarily oriented) cycle; the positive and negative parts of the circuit correspond to the subsets of edges oriented in one or the other direction along this cycle; in particular, $I(H)$ is a positive circuit if and only if $H$ is an oriented cycle;

5. form a cocircuit if and only if $H$ is a minimal (non-necessarily oriented) cut; the positive and negative parts of the cocircuit correspond to the edges in one or the other direction in this cut; in particular, $I(H)$ is a positive cocircuit if and only if $H$ is an oriented cut.

Remark 3.7 The incidence configuration $I(H)$ of a subgraph $H$ of $G$ is the set of vectors of $I(G)$ contained in a $k$-face of $C(G)$ if and only if $H$ has $n-k$ connected components and $G / H$ is acyclic. Thus:

1. The incidence cone $C(G)$ has dimension $n-1$.

2. The incidence cone $C(G)$ is pointed if and only if $G$ is an acyclic directed graph.

3. The facets of $C(G)$ correspond to the complements of the minimal directed cuts in G. Given a minimal directed cut, the characteristic vector of its sink is a normal vector of the corresponding facet.

\subsection{Vertex characterization and face description of the brick polytope}

Let $\mathcal{N}$ be an irreducible sorting network supporting a pseudoline arrangement $\Lambda$. The contact graph $\Lambda^{\#}$ is our main tool to describe the cone of the brick polytope $\Omega(\mathcal{N})$ at the brick vector $\omega(\Lambda)$ :

Theorem 3.8 The cone of the brick polytope $\Omega(\mathcal{N})$ at the brick vector $\omega(\Lambda)$ is precisely the incidence cone $C\left(\Lambda^{\#}\right)$ of the contact graph $\Lambda^{\#}$ of $\Lambda$.

Proof: Assume that $\Lambda^{\prime}$ is obtained from $\Lambda$ by flipping a contact from its $i$ th pseudoline to its $j$ th pseudoline. Then the difference $\omega\left(\Lambda^{\prime}\right)-\omega(\Lambda)$ is a positive multiple of $e_{j}-e_{i}$. This immediately implies that the incidence cone $C\left(\Lambda^{\#}\right)$ is included in the cone of $\Omega(\mathcal{N})$ at $\omega(\Lambda)$.

Reciprocally, we shall prove that any facet $F$ of the cone $C\left(\Lambda^{\#}\right)$ is also a facet of the brick polytope $\Omega(\mathcal{N})$. According to Remark 3.7 (3), there exists a minimal directed cut $\gamma$ of $\Lambda^{\#}$ which partitions the vertices of $\Lambda^{\#}$ between its source $U$ and its sink $V$ such that $\mathbb{1}_{V}:=\sum_{v \in V} e_{v}$ is a normal vector of $F$. We claim that for any pseudoline arrangement $\Lambda^{\prime}$ supported by $\mathcal{N}$, the scalar product $\left\langle\mathbb{1}_{V} \mid \omega\left(\Lambda^{\prime}\right)\right\rangle$ equals $\left\langle\mathbb{1}_{V} \mid \omega(\Lambda)\right\rangle$ when $\gamma$ is a subset of the contacts of $\Lambda^{\prime}$, and is strictly bigger than $\left\langle\mathbb{1}_{V} \mid \omega(\Lambda)\right\rangle$ otherwise. 
The set of all pseudoline arrangements supported by $\mathcal{N}$ whose set of contacts contains $\gamma$ is connected by flips. Since a flip between two such pseudoline arrangements necessarily involves either two pseudolines of $U$ or two pseudolines of $V$, the corresponding incidence vector is orthogonal to $\mathbb{1}_{V}$. Thus, the scalar product $\left\langle\mathbb{1}_{V} \mid \omega\left(\Lambda^{\prime}\right)\right\rangle$ is constant on all pseudoline arrangements whose set of contacts contains $\gamma$.

Reciprocally, since $\gamma$ is a directed cut which separates $U$ and $V$ in the contact graph of $\Lambda$, the crossing between two pseudolines of $\Lambda$ labeled by $u \in U$ and $v \in V$ is positioned as much as possible to the left of the network if $u<v$ and to the right of the network if $u>v$. This implies that the pseudolines labeled by $V$ in any pseudoline arrangement $\Lambda^{\prime}$ supported by $\mathcal{N}$ cover each brick at least as many times as the pseudolines of $\Lambda$ labeled by $V$. Now assume that $\gamma$ is not a subset of the contacts of $\Lambda^{\prime}$, and consider any commutator of $\gamma$ which is not a contact of $\Lambda^{\prime}$. Then one of the brick immediately adjacent to this commutator is covered once more by the pseudolines of $\Lambda^{\prime}$ labeled by $V$ than by the pseudolines of $\Lambda$ labeled by $V$. This implies that $\left\langle\mathbb{1}_{V} \mid \omega\left(\Lambda^{\prime}\right)\right\rangle>\left\langle\mathbb{1}_{V} \mid \omega(\Lambda)\right\rangle$.

This theorem together with Remark 3.7 have the following immediate applications:

Corollary 3.9 The brick polytope of an irreducible sorting network with n levels has dimension $n-1$.

Corollary 3.10 The brick vector $\omega(\Lambda)$ is a vertex of the brick polytope $\Omega(\mathcal{N})$ if and only if $\Lambda^{\#}$ is acyclic.

For example, since their contact graphs are sorted, the source and the sink of the oriented graph of increasing flips always appear as vertices of $\Omega(\mathcal{N})$. These two greedy pseudoline arrangements can be the only vertices of the brick polytope, as happens for 2-level networks.

In general, the application $\omega: \operatorname{Arr}(\mathcal{N}) \rightarrow \mathbb{R}^{n}$ is not injective on $\operatorname{Arr}(\mathcal{N})$ (see e.g. Example 3.16). However, the vertices of the brick polytope have precisely one preimage by $\omega$ :

Proposition 3.11 The application $\omega: \operatorname{Arr}(\mathcal{N}) \rightarrow \mathbb{R}^{n}$ restricts to a bijection between the pseudoline arrangements supported by $\mathcal{N}$ whose contact graphs are acyclic and the vertices of the brick polytope $\Omega(\mathcal{N})$.

Proof: According to Corollary 3.10 , the application $\omega$ defines a surjection from the pseudoline arrangements supported by $\mathcal{N}$ whose contact graphs are acyclic to the vertices of the brick polytope $\Omega(\mathcal{N})$. To prove injectivity, we use an inductive argument based on the following claims: (i) the greedy pseudoline arrangement $\Gamma(\mathcal{N})$ is the unique preimage of $\omega(\Gamma(\mathcal{N}))$; and (ii) if a vertex of $\Omega(\mathcal{N})$ has a unique preimage by $\omega$, then so do its neighbors in the graph of $\Omega(\mathcal{N})$.

To prove (i), consider a pseudoline arrangement $\Lambda$ supported by $\mathcal{N}$ such that $\omega(\Lambda)=\omega(\Gamma(\mathcal{N}))$. According to Theorem 3.8 , the contact graphs $\Lambda^{\#}$ and $\Gamma(\mathcal{N})^{\#}$ have the same incidence cone, which ensures that all $\operatorname{arcs}$ of $\Lambda^{\#}$ are sorted. In other words, all flips in $\Lambda$ are increasing. Since this property characterizes the greedy pseudoline arrangement, we obtain that $\Lambda=\Gamma(\mathcal{N})$.

To prove (ii), consider two neighbors $v, v^{\prime}$ in the graph of $\Omega(\mathcal{N})$. Let $i, j \in[n]$ and $\alpha>0$ be such that $v^{\prime}-v=\alpha\left(e_{j}-e_{i}\right)$. Let $\Lambda$ be a pseudoline arrangement supported by $\mathcal{N}$ such that $v=\omega(\Lambda)$. Let $\Lambda^{\prime}$ denote the pseudoline arrangement obtained from $\Lambda$ by flipping the rightmost contact between its $i$ th and $j$ th pseudolines if $i<j$ and the leftmost one if $i>j$. Then $v^{\prime}=\omega\left(\Lambda^{\prime}\right)$. In particular, if $v$ has two distinct preimages by $\omega$, then so does $v^{\prime}$. This proves (ii).

Corollary 3.12 The graph of the brick polytope is a subgraph of $G(\mathcal{N})$ whose vertices are the pseudoline arrangements with acyclic contact graphs. However, note that the graph of the brick polytope is not always the subgraph of $G(\mathcal{N})$ induced by the pseudoline arrangements with acyclic contact graphs. 
Remark 3.7 (3) and Theorem 3.8 also provides the normal vectors of the brick polytope:

Corollary 3.13 The facet normal vectors of the brick polytope $\Omega(\mathcal{N})$ are precisely all normal vectors of the incidence cones of the contact graphs of the pseudoline arrangements supported by $\mathcal{N}$. Representative for them are given by the characteristic vectors of the sinks of the minimal directed cuts of these graphs.

More generally, Theorem 3.8, Remark 3.7, and Proposition 3.11 provide a combinatorial description of the faces of the brick polytope. We need the following definition:

Definition 3.14 A set $\gamma$ of commutators of $\mathcal{N}$ is $k$-admissible if there exists a pseudoline arrangement $\Lambda \in \operatorname{Arr}(\mathcal{N})$ whose set of contacts contain $\gamma$ and such that $\Lambda^{\#} \backslash \gamma^{\#}$ has $n-k$ connected components and $\Lambda^{\#} /\left(\Lambda^{\#} \backslash \gamma^{\#}\right)$ is acyclic (where $\gamma^{\#}$ is the subgraph of $\Lambda^{\#}$ corresponding to the commutators of $\gamma$ ).

Corollary 3.15 Let $\Phi$ be the application which associates to a subset $X$ of $\mathbb{R}^{n}$ the set of commutators of $\mathcal{N}$ which are contacts in all the pseudoline arrangements supported by $\mathcal{N}$ whose brick vectors lie in $X$. Let $\Psi$ be the application which associates to a set $\gamma$ of commutators of $\mathcal{N}$ the convex hull of the brick vectors of the pseudoline arrangements supported by $\mathcal{N}$ whose set of contacts contains $\gamma$. Then the applications $\Phi$ and $\Psi$ define inverse bijections between the $k$-faces of $\Omega(\mathcal{N})$ and the $k$-admissible sets of commutators of $\mathcal{N}$.

Example 3.16 (Duplicated networks) Consider a network $\Pi$ with $n$ levels and $2\left(\begin{array}{c}n \\ 2\end{array}\right)$ commutators obtained by duplicating each commutator of a sorting network $\bar{\Pi}$ with $n$ levels and $\left(\begin{array}{c}n \\ 2\end{array}\right)$ commutators. Any pseudoline arrangement $\Lambda$ supported by $\Pi$ has one contact and one crossing among each pair of adjacent commutators of $\Pi$, and the contact graph $\Lambda^{\#}$ is a tournament (an oriented complete graph). Thus, the vertices of the brick polytope $\Omega(\Pi)$ correspond to the permutations of $[n]$, representatives of its facet normal vectors are given by the vectors of $\{0,1\}^{n}$ except $0^{n}$ and $1^{n}$, and its $k$-faces are in one-to-one correspondence with ordered $(n-k)$-partitions of $[n]$. The brick polytope $\Omega(\Pi)$ is a permutahedron. It is in fact a translate of the usual permutahedron $P_{n}:=\operatorname{conv}\left\{(\sigma(1), \ldots, \sigma(n))^{T} \mid \sigma \in \mathfrak{S}_{n}\right\}$.

To finish this section, we apply our results to minimal sorting networks (i.e. such that the contact graphs of the pseudoline arrangements they support are trees — see Definition 3.4.

Theorem 3.17 For any minimal irreducible sorting network $\mathcal{N}$, the simplicial complex $\Delta(\mathcal{N})$ is isomorphic to the boundary complex of the polar of the brick polytope $\Omega(\mathcal{N})$. In particular, the graph of $\Omega(\mathcal{N})$ is isomorphic to the flip graph $G(\mathcal{N})$.

Proof: Since any orientation on a tree is acyclic, any set $\gamma$ of commutators of $\mathcal{N}$ such that $\mathcal{N} \backslash \gamma$ is sorting is admissible. Thus, the boundary complex of $\Omega(\mathcal{N})$ is isomorphic to $\Delta(\mathcal{N})$ via Corollary 3.15 .

\subsection{Brick polytopes and Minkowski decompositions}

Let $\mathcal{N}$ be a sorting network with $n$ levels and let $b$ be a brick of $\mathcal{N}$. For any pseudoline arrangement $\Lambda$ supported by $\mathcal{N}$, we denote by $\omega(\Lambda, b) \in \mathbb{R}^{n}$ the characteristic vector of the pseudolines of $\Lambda$ passing above $b$. We define the polytope $\Omega(\mathcal{N}, b):=\operatorname{conv}\{\omega(\Lambda, b) \mid \Lambda \in \operatorname{Arr}(\mathcal{N})\} \subset \mathbb{R}^{n}$. Note that the vertex set of $\Omega(\mathcal{N}, b)$ is contained in the vertex set of an hypersimplex, since the number of pseudolines above $b$ always equals the depth of $b$. These polytopes decompose $\Omega(\mathcal{N})$ into a Minkowski sum:

Proposition 3.18 The brick polytope $\Omega(\mathcal{N})$ is the Minkowski sum of the polytopes $\Omega(\mathcal{N}, b)$ associated to all the bricks $b$ of $\mathcal{N}$. 
Proof: Since $\omega(\Lambda)=\sum \omega(\Lambda, b)$ for any pseudoline arrangement $\Lambda$ supported by $\mathcal{N}$, the brick polytope $\Omega(\mathcal{N})$ is included in the Minkowski sum $\sum \Omega(\mathcal{N}, b)$. To prove equality, we thus only have to prove that any vertex of $\sum \Omega(\mathcal{N}, b)$ is indeed a vertex of $\Omega(\mathcal{N})$.

Let $f: \mathbb{R}^{n} \rightarrow \mathbb{R}$ be a linear function, and $\Lambda, \Lambda^{\prime}$ be two pseudoline arrangements related by a flip involving their $i$ th and $j$ th pseudolines. If a brick $b$ of $\mathcal{N}$ is not located between the $i$ th pseudolines of $\Lambda$ and $\Lambda^{\prime}$, then $f(\omega(\Lambda, b))=f\left(\omega\left(\Lambda^{\prime}, b\right)\right)$. Otherwise, the variation $f(\omega(\Lambda, b))-f\left(\omega\left(\Lambda^{\prime}, b\right)\right)$ has the same sign as the variation $f(\omega(\Lambda))-f\left(\omega\left(\Lambda^{\prime}\right)\right)$. Consequently, the pseudoline arrangement $\Lambda_{f}$ supported by $\mathcal{N}$ which minimizes $f$ on $\Omega(\mathcal{N})$, also minimizes $f$ on $\Omega(\mathcal{N}, b)$ for each brick $b$ of $\mathcal{N}$.

Now let $v$ be any vertex of $\sum \Omega(\mathcal{N}, b)$. Let $f: \mathbb{R}^{n} \rightarrow \mathbb{R}$ denote a linear function which is minimized by $v$ on $\sum \Omega(\mathcal{N}, b)$. Then $v$ is the sum of the vertices which minimize $f$ in each summand $\Omega(\mathcal{N}, b)$. Consequently, we obtain that $v=\sum \omega\left(\Lambda_{f}, b\right)=\omega\left(\Lambda_{f}\right)$ is a vertex of $\Omega(\mathcal{N})$.

Example 3.19 (Duplicated networks, continued) A duplicated network $\Pi$ (see Example 3.16) has two types of bricks: those located between two adjacent commutators (which replace the commutators of $\bar{\Pi}$ ) and the other ones (which correspond to the bricks of $\bar{\Pi})$. For any brick $b$ of the later type, the polytope $\Omega(\Pi, b)$ is still a single point. Now let b be a brick of $\Pi$ located between two adjacent commutators. Let $i, j$ be such that $b$ replaces the crossing of the ith and $j$ th pseudolines of the pseudoline arrangement supported by $\bar{\Pi}$. Then $\Omega(\Pi, b)$ is (a translate of) the segment $\left[e_{i}, e_{j}\right]$. Summing the contributions of all bricks, we obtain that the permutahedron $\Omega(\Pi)$ is the Minkowski sum of all segments $\left[e_{i}, e_{j}\right]$ for $1 \leq i<j \leq n$.

Another Minkowski decomposition of our brick polytopes can be derived from the study of the wellbehaved class of generalized permutahedra developed by Postnikov (2009) and Ardila et al. (2010):

Definition 3.20 (Postnikov (2009)) A generalized permutohedra is a polytope with inequality description:

$$
\mathbb{Z}\left(\left\{z_{I}\right\}_{I \in[n]}\right):=\left\{\left(x_{1}, \ldots, x_{n}\right)^{T} \in \mathbb{R}^{n} \mid \sum_{i=1}^{n} x_{i}=z_{[n]} \text { and } \sum_{i \in I} x_{i} \geq z_{I} \text { for } I \subset[n]\right\}
$$

for some family $\left\{z_{I}\right\}_{I \subset[n]} \in \mathbb{R}^{2^{[n]}}$.

Since $\mathbb{Z}\left(\left\{z_{I}\right\}\right)+\mathbb{Z}\left(\left\{z_{I}^{\prime}\right\}\right)=\mathbb{Z}\left(\left\{z_{I}+z_{I}^{\prime}\right\}\right)$, the class of generalized associahedra is closed by Minkowski sum and difference (a Minkowski difference $P-Q$ of two polytopes $P, Q \subset \mathbb{R}^{n}$ is defined only if there exists a polytope $R$ such that $P=Q+R$ ). Consequently, for any $\left\{y_{I}\right\}_{I \subset[n]} \in \mathbb{R}^{2^{[n]}}$, the Minkowski sum and difference $\mathbb{Y}\left(\left\{y_{I}\right\}_{I \in[n]}\right):=\sum_{I \subset[n]} y_{I} \triangle_{I}$ of faces $\triangle_{I}:=\operatorname{conv}\left\{e_{i} \mid i \in I\right\}$ of the standard simplex $\triangle_{[n]}$ is a generalized permutahedron. Reciprocally, it turns out that any generalized permutahedron $\mathbb{Z}\left(\left\{z_{I}\right\}\right)$ can be decomposed as such a Minkowski sum and difference $\mathbb{Y}\left(\left\{y_{I}\right\}\right)$, and that $\left\{y_{I}\right\}$ is derived from $\left\{z_{I}\right\}$ by a simple inversion formula when all the inequalities defining $\mathbb{Z}\left(\left\{z_{I}\right\}\right)$ are tight:

Proposition 3.21 (Postnikov (2009), Ardila et al. (2010)) Every generalized permutahedron can be written uniquely as a Minkowski sum and difference of faces of the standard simplex: $\mathbb{Z}\left(\left\{z_{I}\right\}\right)=\mathbb{Y}\left(\left\{y_{I}\right\}\right)$, where $y_{I}=\sum_{J \subset I}(-1)^{|I \backslash J|} z_{J}$ if all inequalities $\sum_{i \in I} x_{i} \geq z_{I}$ defining $\mathbb{Z}\left(\left\{z_{I}\right\}\right)$ are tight.

Example 3.22 The classical permutahedron can be written as $\Pi_{n}=\mathbb{Z}\left(\left\{\frac{|I|(|I|+1)}{2}\right\}\right)=\sum_{|I|=2} \triangle_{I}$.

The Minkowski decomposition of Proposition 3.21 is used by Postnikov (2009) to compute the volume of the generalized permutohedra.

According to Lemma 2.3 and Corollary 3.13 , all our brick polytopes are generalized permutohedra. It raises the question to compute efficiently their Minkowski decomposition into dilates of faces of the standard simplex. This question was recently addressed by Lange (2011) for the special sorting networks whose brick polytopes are associahedra and which we present in the next section. 


\section{Hohlweg and Lange's associahedra, revisited}

Based on the duality between triangulations of a convex polygon and pseudoline arrangements supported by the 1-kernel of a reduced alternating sorting network, we observe in this section that the brick polytopes of these particular networks specialize to the associahedra of Hohlweg and Lange (2007).

\subsection{Duality}

We call reduced alternating sorting network any network with $n$ levels and $\left(\begin{array}{l}n \\ 2\end{array}\right)$ commutators such that the commutators adjacent to each intermediate level are alternatively located above and below it. Such a network supports a unique pseudoline arrangement, whose first and last pseudolines both touch its top and its bottom level, and whose intermediate pseudolines all touch either its top or its bottom level.

To a word $x \in\{a, b\}^{n-2}$, we associate the following two dual objects — see Figure 4 (left):

- $\mathcal{P}_{x}$ denotes the $n$-gon obtained as the convex hull of $\left\{p_{i} \mid i \in[n]\right\}$ where $p_{1}=(1,0), p_{n}=(n, 0)$ and $p_{i+1}$ is the point of the circle of diameter $\left[p_{1}, p_{n}\right]$ with abscissa $i+1$ and located above $\left[p_{1}, p_{n}\right]$ if $x_{i}=a$ and below $\left[p_{1}, p_{n}\right]$ if $x_{i}=b$, for all $i \in[n-2]$.

- $\mathcal{N}_{x}$ denotes the reduced alternating sorting network such that the $(i+1)$ th pseudoline touches its top level if $x_{i}=a$ and its bottom level if $x_{i}=b$, for all $i \in[n-2]$.

For any $1 \leq i<j \leq n$, we naturally both label by $(i, j)$ the diagonal $\left[p_{i}, p_{j}\right]$ of $\mathcal{P}_{x}$ and the commutator of $\mathcal{N}_{x}$ where cross the $i$ th and $j$ th pseudolines of the unique pseudoline arrangement supported by $\mathcal{N}_{x}$.
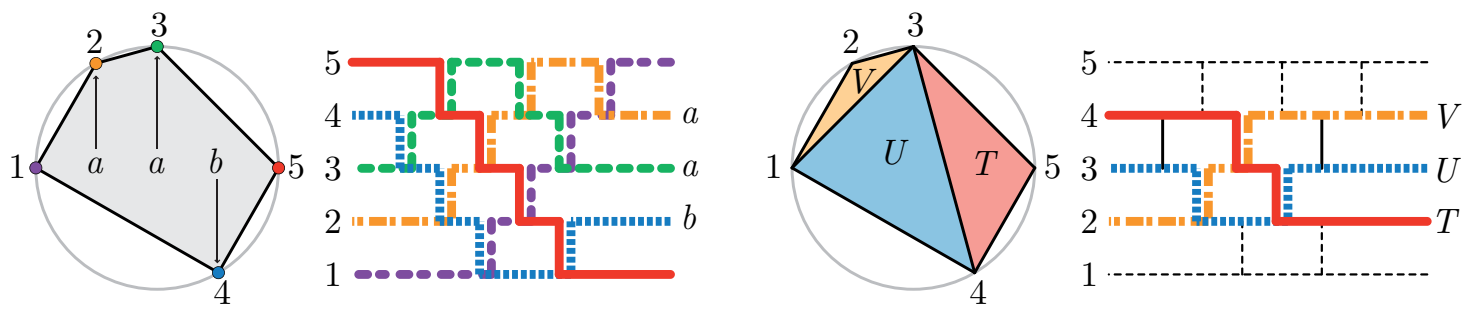

Fig. 4: The polygon $\mathcal{P}_{a a b}$ and the network $\mathcal{N}_{a a b}$ (left). A triangulation $T$ of $\mathcal{P}_{a a b}$ and its dual $T^{*}$ on $\mathcal{N}_{a a b}$ (right).

We call 1-kernel of a network $\mathcal{N}$ the network $\mathcal{N}^{1}$ obtained from $\mathcal{N}$ by erasing its first and last horizontal lines, as well as all commutators incident to them. For a word $x \in\{a, b\}^{n-2}$, the network $\mathcal{N}_{x}^{1}$ has $n-2$ levels and $\left(\begin{array}{l}n \\ 2\end{array}\right)-n$ commutators. Since we erased the commutators between consecutive pseudolines on the top or bottom level of $\mathcal{N}_{x}$, the remaining commutators are labeled by the internal diagonals of $\mathcal{P}_{x}$.

Proposition 4.1 (Pilaud and Pocchiola (2010)) Fix $a$ word $x \in\{a, b\}^{n-2}$. The set of commutators of $\mathcal{N}_{x}$ labeled by the internal diagonals of a triangulation $T$ of $\mathcal{P}_{x}$ is the set of contacts of a pseudoline arrangement $T^{*}$ supported by $\mathcal{N}_{x}^{1}$. Reciprocally the internal diagonals of $\mathcal{P}_{x}$ which label the contacts of a pseudoline arrangement supported by $\mathcal{N}_{x}^{1}$ form a triangulation of $\mathcal{P}_{x}$.

The dual pseudoline arrangement $T^{*}$ of a triangulation $T$ of $\mathcal{P}_{x}$ has one pseudoline $\Delta^{*}$ dual to each triangle $\Delta$ of $T$. A commutator is the crossing (resp. a contact) between two pseudolines $\Delta^{*}$ and $\Delta^{\prime *}$ 
of $T^{*}$ if it is labeled by the common bisector (resp. by a common edge) of the triangles $\Delta$ and $\Delta^{\prime}$. Consequently, this duality defines an isomorphism between the graph of flips on pseudoline arrangements supported by $\mathcal{N}_{x}^{1}$ and the graph of flips on triangulations of $\mathcal{P}_{x}$. Furthermore, we obtain the following interpretation of the contact graph of $T^{*}$ :

Lemma 4.2 The contact graph $\left(T^{*}\right)^{\#}$ of the dual pseudoline arrangement $T^{*}$ of a triangulation $T$ is precisely the dual tree of $T$, with some additional orientations on the edges.

Remark 4.3 This duality has been extended by Pilaud and Pocchiola (2010) in two different directions: on the one hand, to pseudotriangulations of point sets in general position (see Rote et al. (2008)), and on the other hand, to multitriangulations of convex polygon (see Pilaud and Santos (2009)). We refer to the work of Pilaud and Pocchiola (2010) or to Chapter 3 of Pilaud (2010) for precise definitions and details.

\subsection{Associahedra}

Consider a reduced alternating sorting network $\mathcal{N}_{x}$ with $n$ levels. According to Proposition 4.1 and Lemma 4.2 its 1-kernel $\mathcal{N}_{x}^{1}$ is a minimal network: the pseudoline arrangements it supports correspond to triangulations of $\mathcal{P}_{x}$ and their contact graphs are the dual trees of these triangulations. Thus, we obtain:

Proposition 4.4 For any $x \in\{a, b\}^{n-2}$, the simplicial complex of crossing-free sets of internal diagonals of the convex $n$-gon $\mathcal{P}_{x}$ is (isomorphic to) the boundary complex of the polar of the brick polytope $\Omega\left(\mathcal{N}_{x}^{1}\right)$. Thus, the brick polytope $\Omega\left(\mathcal{N}_{x}^{1}\right)$ is a realization of the $(n-3)$-dimensional associahedron.

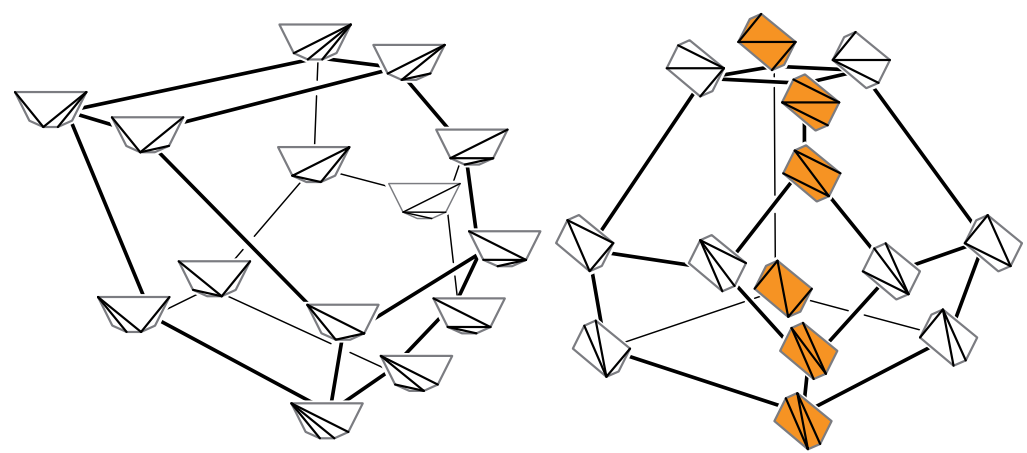

Fig. 5: The brick polytopes $\Omega\left(\mathcal{N}_{b^{4}}^{1}\right)$ (left) and $\Omega\left(\mathcal{N}_{a^{2} b^{2}}^{1}\right)$ (right) provide two different realizations of the 3 -dimensional associahedron. The convex hull of the brick vectors of the centrally symmetric triangulations of $\mathcal{P}_{a^{2} b^{2}}$ (colored in the picture) is a realization of the 2-dimensional cyclohedron.

Up to translation, our brick polytopes are precisely the associahedra of Hohlweg and Lange (2007). In their presentation, the associahedron is obtained as the convex hull of vectors directly associated to each triangulation of the polygon $\mathcal{P}_{x}$.

Observe that the associahedron $\Omega\left(\mathcal{N}_{x}^{1}\right)$ does not depend on the first and last letters of $x$ since we erase the first and last levels of $\mathcal{N}_{x}$. Furthermore, a network $\mathcal{N}_{x}$ and its reflection through the vertical (resp. horizontal) axis give rise to affinely equivalent associahedra. Affine equivalence between these associahedra is studied by Bergeron et al. (2009). Two non-affinely equivalent 3-dimensional associahedra are presented in Figure 5 
Example 4.5 The brick polytope of the 1-kernel of the "bubble sort" network $\mathcal{B}_{n}:=\mathcal{N}_{b^{n-2}}$ coincides (up to translation) with the $(n-3)$-dimensional associahedron of Loday (2004) - see Figure 5 (left).

We now describe the normal vectors of the facets of our associahedra. For any $x \in\{a, b\}^{n-2}$, the facets of the brick polytope $\Omega\left(\mathcal{N}_{x}^{1}\right)$ are in bijection with the commutators of $\mathcal{N}_{x}^{1}$. The vertices of the facet corresponding to a commutator $\gamma$ are the brick vectors of the pseudoline arrangements supported by $\mathcal{N}_{x}^{1}$ and with a contact at $\gamma$. We have already seen that a normal vector of this facet is given by the characteristic vector of the sink of the cut induced by $\gamma$ in the contact graphs of the pseudoline arrangements supported by $\mathcal{N}_{x}^{1}$ and with a contact at $\gamma$. In the following lemma, we give an additional description of this characteristic vector:

Lemma 4.6 Let $\Lambda$ be a pseudoline arrangement supported by $\mathcal{N}_{x}^{1}$ and let $\gamma$ be a contact of $\Lambda$. The arc corresponding to $\gamma$ is a cut of the contact graph $\Lambda^{\#}$ which separates the pseudolines of $\Lambda$ passing above $\gamma$ from those passing below $\gamma$.

Corollary 4.7 For any $x \in\{a, b\}^{n-2}$, the brick polytope $\Omega\left(\mathcal{N}_{x}^{1}\right)$ has $n-3$ pairs of parallel facets. The diagonals of $\mathcal{P}_{x}$ corresponding to two parallel facets of $\Omega\left(\mathcal{N}_{x}^{1}\right)$ are crossing.

Remark 4.8 As discussed in Section 3.4 the associahedron $\Omega\left(\mathcal{N}_{x}^{1}\right)$ has two different Minkowski decompositions: as a positive Minkowski sum $\sum_{b} \Omega\left(\mathcal{N}_{x}^{1}, b\right)$ of the polytopes $\Omega\left(\mathcal{N}_{x}^{1}, b\right)$ associated to each brick $b$ of $\mathcal{N}_{x}^{1}$, or as a Minkowski sum and difference $\sum_{I \subset[n-2]} y_{I} \triangle_{I}$ offaces $\triangle_{I}$ of the standard simplex $\triangle_{[n-2]}$.

In Loday's associahedron (i.e. when $x=b^{n-2}$ and $\mathcal{N}_{x}:=\mathcal{B}_{n}$ ), these two decompositions coincide. Indeed, for any $i, j \in[n]$ with $j \geq i+3$, denote by $b(i, j)$ the brick of $\mathcal{B}_{n}^{1}$ located immediately below the contact between the ith and the jth pseudoline of the unique pseudoline arrangement supported by $\mathcal{B}_{n}$. Then the Minkowski summand $\Omega\left(\mathcal{B}_{n}^{1}, b(i, j)\right)$ is the face $\triangle_{\{i, \ldots, j-2\}}$ of the standard simplex (up to a translation of vector $\left.\mathbb{1}_{\{1, \ldots, i-1\} \cup\{j-1, \ldots, n-2\}}\right)$. This implies that $\Omega\left(\mathcal{B}_{n}^{1}\right)=\sum_{1 \leq i<j \leq n-2} \triangle_{\{i, \ldots, j\}}$.

For general $x$, the Minkowski summands $\Omega\left(\mathcal{N}_{x}^{1}, b\right)$ are not always simplices. A recent work of Lange (2011) describes the coefficients $\left\{y_{I}\right\}$ in the Minkowski decomposition of any associahedra $\Omega\left(\mathcal{N}_{x}^{1}\right)$.

Remark 4.9 To close this section, we want to mention that we can similarly present Hohlweg \& Lange's realizations of the cyclohedra. Namely, consider a antisymmetric word $x \in\{a, b\}^{2 n-2}$ (i.e. which satisfies $\left\{x_{i}, x_{2 n-1-i}\right\}=\{a, b\}$ for all $\left.i\right)$, such that the $(2 n)$-gon $\mathcal{P}_{x}$ is centrally symmetric. Then the convex hull of the brick vectors of the dual pseudoline arrangements of all centrally symmetric triangulations of $\mathcal{P}_{x}$ is a realization of the $(n-1)$-dimensional cyclohedron. For example, the centrally symmetric triangulations of $\mathcal{P}_{a^{2} b^{2}}$ are colored in the right associahedron of Figure 5 the convex hull of the corresponding vertices is a realization of the 2-dimensional cyclohedron.

\section{Conclusion}

We associated a brick vector to each pseudoline arrangement supported by a given network $\mathcal{N}$. The convex hull of these vectors, the brick polytope, has an interesting combinatorial structure: its graph is a subgraph of the flip graph $G(\mathcal{N})$ and it generalizes Hohlweg and Lange's associahedra.

This paper was originally motivated by the question of the polytopality of the simplicial complex $\Delta(\mathcal{N})$. We could expect our brick polytope to be a first step towards this question, in the sense that $\Omega(\mathcal{N})$ could be a projection of the polar of a realization of $\Delta(\mathcal{N})$. However, this is false in general. More details on this question will appear in a long version of this paper (see Pilaud and Santos (2010)). 


\section{References}

F. Ardila, C. Benedetti, and J. Doker. Matroid polytopes and their volumes. Discrete Comput. Geom., 43 (4):841-854, 2010.

N. Bergeron, C. Hohlweg, C. Lange, and H. Thomas. Isometry classes of generalized associahedra. Séminaire Lotharingien de Combinatoire, B61Aa, 2009.

L. J. Billera, P. Filliman, and B. Sturmfels. Constructions and complexity of secondary polytopes. Adv. Math., 83(2):155-179, 1990.

A. Björner, M. L. Vergnas, B. Sturmfels, N. White, and G. M. Ziegler. Oriented matroids, volume 46 of Encyclopedia of Mathematics and its Applications. Cambridge University Press, Cambridge, second edition, 1999.

J. A. De Loera, J. Rambau, and F. Santos. Triangulations: Structures for Algorithms and Applications, volume 25 of Algorithms and Computation in Mathematics. Springer Verlag, 2010.

I. M. Gel'fand, M. M. Kapranov, and A. V. Zelevinsky. Discriminants, resultants, and multidimensional determinants. Mathematics: Theory \& Applications. Birkhäuser Boston Inc., Boston, MA, 1994.

C. Hohlweg and C. E. M. C. Lange. Realizations of the associahedron and cyclohedron. Discrete Comput. Geom., 37(4):517-543, 2007.

A. Knutson and E. Miller. Subword complexes in Coxeter groups. Adv. Math., 184(1):161-176, 2004.

C. Lange. Minkowski decompositions of associahedra. Preprint (20 pages). Extended abstract in $23 \mathrm{rd}$ International Conference on Formal Power Series and Algebraic Combinatorics, 2011.

C. W. Lee. The associahedron and triangulations of the n-gon. European J. Combin., 10(6):551-560, 1989.

J.-L. Loday. Realization of the Stasheff polytope. Arch. Math. (Basel), 83(3):267-278, 2004.

V. Pilaud. Multitriangulations, pseudotriangulations and some problems of realization of polytopes. $\mathrm{PhD}$ thesis, Université Paris 7 \& Universidad de Cantabria, 2010.

V. Pilaud and M. Pocchiola. Multitriangulations, pseudotriangulations and primitive sorting networks. Preprint, arXiv:1009.5344. Extended abstract in 25th European Workshop on Comput. Geom. (Bruxelles), 2010.

V. Pilaud and F. Santos. Multitriangulations as complexes of star polygons. Discrete Comput. Geom., 41 (2):284-317, 2009.

V. Pilaud and F. Santos. The brick polytope of a sorting network. Preprint, arXiv: 1103.2731, 2010.

A. Postnikov. Permutohedra, associahedra, and beyond. Int. Math. Res. Not. IMRN, (6):1026-1106, 2009.

G. Rote, F. Santos, and I. Streinu. Pseudo-triangulations - a survey. In Surveys on discrete and computational geometry, volume 453 of Contemp. Math., pages 343-410. Amer. Math. Soc., Providence, RI, 2008. 